\title{
Мощные оптоэлектронные коммутаторы нано- и пикосекундного диапазона на основе высоковольтных кремниевых структур с $p-n$-переходами. І. Физика процесса переключения
}

\author{
(C) A.C. Кюрегян \\ Всероссийский электротехнический институт им. В.И. Ленина, \\ 111250 Москва, Россия \\ E-mail: semlab@yandex.ru
}

(Получена 21 декабря 2016 г. Принята к печати 28 февраля 2017 г.)

Впервые проведено численное моделирование процесса переключения высоковольтных кремниевых фотодиодов, фототранзисторов и фототиристоров под действием квазиоднородного по площади освещения пикосекундными лазерными импульсами. Анализ результатов позволил получить „эмпирические“ соотношения между основными параметрами коммутаторов (энергией управляющих импульсов, коэффициентом поглощения излучения, площадью структур) и параметрами, характеризующими переходный процесс переключения в цепи с активной нагрузкой. Для некоторых из этих соотношений выведены приближенные аналитические формулы, хорошо описывающие результаты моделирования. Отмечено, что различия между процессами коммутации в трех типах структур проявляются только при больших длительностях импульсов на заключительной стадии, когда восстанавливается блокирующая способность фотодиодов и фототранзисторов.

DOI: $10.21883 /$ FTP.2017.09.44891.8494

\section{1. Введение}

Попытки использования управляемых светом структур с $p-n$-переходами для формирования мощных высоковольтных электрических импульсов с наносекундными фронтами продолжаются уже более 40 лет [1-11]. Однако до сих пор такие коммутаторы не нашли заметного применения. Длительное время развитие этого направления импульсной техники сдерживалось низкими эксплуатационными характеристиками (такими как энергетическая эффективность, надежность, массогабаритные показатели, стоимость) импульсных источников света (Nd-YAG лазеров), необходимых для управления высоковольтными кремниевыми приборами. Создание и совершенствование в течение последних 20 лет волоконных лазеров с диодной накачкой практически разрешило эту проблему, ${ }^{1}$ но для разработки высокоэффективных оптоэлектронных коммутаторов необходимо еще ясное понимание физики процесса переключения, определяющей сложную нелинейную взаимосвязь между параметрами нагрузки, полупроводниковых структур и управляющих импульсов света. Вместе тем опубликованные экспериментальные работы содержат лишь отрывочные сведения и имеют, скорее, демонстрационный, нежели исследовательский характер, а аналитические теории [13-15] не учитывают всю совокупность нелинейных эффектов, определяющих переходные характеристики структур вследствие неоднородности их легирования, зависимости подвижности от напряженности поля и ударной ионизации. В настоящей работе изложены

\footnotetext{
${ }^{1}$ Сейчас доступны комерческие высоконадежные импульсные источники излучения с практическим кпд $>20 \%$, длительностью импульса $0.01-10$ нс, энергией $0.01-10$ мДж и длиной волны $\sim 1064$ нм, почти идеально подходящей для управления высоковольтными кремниевыми приборами (например, волоконные лазеры фирмы IPG-photonics [12]).
}

результаты систематического исследования физических особенностей переключения таких приборов путем численного моделирования процесса переключения.

\section{2. Объекты и метод исследования}

Моделировался процесс переключения структур типа $p^{+}-p-v-n-n^{+}$(фотодиодов), $p^{+}-p-v-n-p^{+}$(фототранзисторов) и $n^{++}-p^{+}-p-v-n-p^{+}$(фототиристоров) из блокирующего состояния при напряжении $U_{0}=5$ кВ в проводящее состояние. Конструкция приборов схематически изображена на рис. 1. Кремниевые структуры соединены с металлическими электродами. В катодном электроде имеется $m$ отверстий площадью $S_{1} \sim 1$ мм $^{2}$, совмещенных с окнами в металлизации структур, сквозь которые проходит излучение лазера.

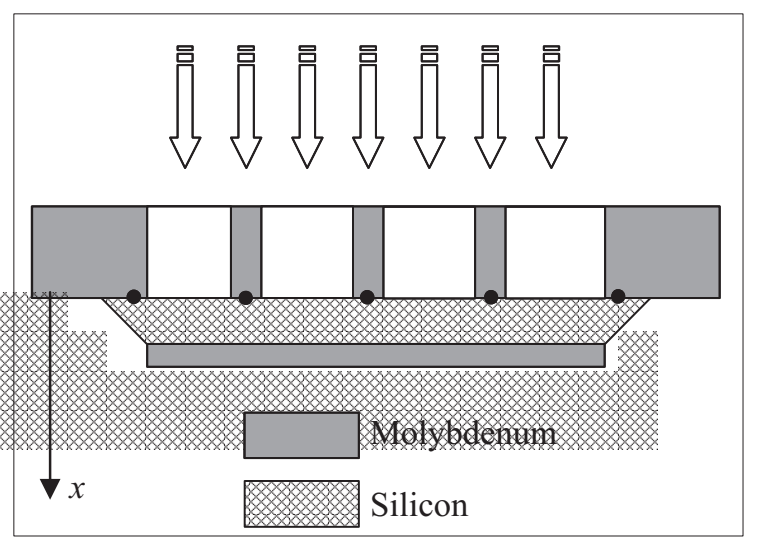

Рис. 1. Схематическое изображение поперечного сечения изучаемых полупроводниковых структур. Точками отмечено положение шунтов на поверхности катода фототиристоров. 


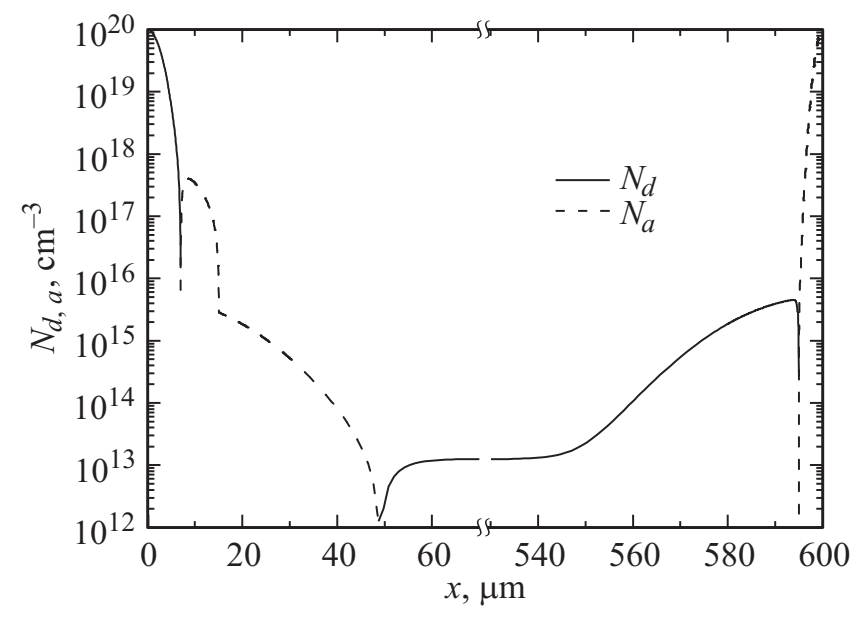

Рис. 2. Распределение концентраций доноров $N_{d}$ (сплошная линия) и акцепторов $N_{a}$ (штриховая линия) по толщине $x$ фототиристора.

Предполагалось, что полная площадь структур есть $S_{D}=2 S_{\mathrm{ph}}$, освещаемая площадь $S_{\mathrm{ph}}=m S_{1}$ изменялась в пределах $0.1-3 \mathrm{~cm}^{2}$. Толщина структур $d=600$ мкм, параметры рекомбинации в модели Шокли-Рида $\tau_{n 0}=\tau_{p 0}=3$ мкс, концентрация доноров в $\nu$-слое $N_{0}=1.25 \cdot 10^{13} \mathrm{~cm}^{-3}$, распределения примесей в диффузионных слоях описывались функцией Гаусса. Использованный нами профиль легирования фототиристоpa $N_{d, a}(x)$, изображенный на рис. 2 , обеспечивал при наличии кольцевой шунтировки котодного $n^{+}-p$-перехода на периферии освещаемых окон и температуре $375 \mathrm{~K}$ напряжение пробоя $U_{B}=6$ кВ при скорости нарастания напряжения до 1 кВ/мкс. Он отличается от предложенного в [11] профиля тем, что прилегающие к $v$-слою диффузионные $p$-база и буферный $n$-слой в $\sim 10$ раз толще и в $\sim 1000$ раз слабее легированы. Это сделано для уменьшения напряженности краевого поля в реальных структурах и подавления динамического лавинного пробоя на первом этапе процесса коммутации (см. далее). Структуры фототранзистора или фотодиода различались тем, что изменялся тип проводимости одного или обоих приэлектродных сильно легированных слоев, а шунтировка отсутствовала. Их напряжения пробоя были равны 6 и $6.6 \mathrm{\kappa B}$ соответственно.

Мощность излучения лазера изменялась со временем по закону $P(t)=P_{p} F_{t}(t)$, где

$$
F_{t}(t)=\sqrt{2 e} \frac{t}{t_{\mathrm{ph}}} \exp \left[-\left(\frac{t}{t_{\mathrm{ph}}}\right)^{2}\right],
$$

$P_{p}$ - пиковая мощность в момент времени $t=t_{\mathrm{ph}} / \sqrt{2}$. При этом энергия импульса излучения $W_{\mathrm{ph}}=\sqrt{2 / e} P_{p} t_{\mathrm{ph}}$. Скорость генерации электронно-дырочных пар в структуре представлялась в виде $G(x, t)=G_{0} F_{t}(t) F_{x}(x)$, где $G_{0}=P_{p} \kappa\left(1-R_{\mathrm{ph}}\right) / \hbar \omega S_{\mathrm{ph}}, \hbar \omega-$ энергия кванта, $\kappa-$ коэффициент поглощения света в полупроводнике, $R_{\mathrm{ph}}$ - коэффициент отражения света от поверхности окна фотодиода. В настоящей работе мы считали, что на поверхность окон нанесено просветляющее покрытие и поэтому $R_{\mathrm{ph}}=0$. Распределение скорости генерации по толщине структур описывалось функцией

$$
F_{x}(x)=2 e^{-\kappa d} \operatorname{ch}[\kappa(d-x)] .
$$

При этом мы пренебрегали относительно слабой зависимостью $\kappa$ от $x$ вследствие эффекта Франца-Келдыша и сужения запрещенной зоны при высоком уровне легирования и считали тыльный контакт (плоскость $x=d$ ) идеально отражающим.

Динамика электронно-дырочной плазмы в структурах моделировалась с помощью программы „Исследование $[16]$ при начальном напряжении на них $U_{0}=5$ кВ и температуре $75^{\circ} \mathrm{C}$. В настоящей работе изучался простейший режим, соответствующий разряду формирующей линии длиной $l$ с волновым сопротивлением $Z$ на согласованную активную нагрузку $R_{L}=Z$. В этом случае через кремниевую структуру и нагрузку протекает импульс тока с длительностью $2 l c$ и амлитудой $\left(U_{0}-U_{\min }\right) / R$, где $c-$ скорость света в диэлектрике, заполняющем линию, $R=2 R_{L}, U_{\min }-$ минимальное падение напряжения на структуре. Далее изложены результаты, полученные при $t_{\mathrm{ph}}=10$ пс для значений $R_{L}=5$ Ом и, если это особо не оговорено, $S_{\mathrm{ph}}=0.5 \mathrm{~cm}^{2}$, $\kappa=32 \mathrm{~cm}^{-1}$ (согласно [17] примерно такое значение $\kappa$ имеет излучение с длиной волны 1045 нм, типичной для волоконных лазеров, в $\mathrm{Si}$ при $\left.75^{\circ} \mathrm{C}\right)$.

\section{3. Результаты моделирования и их обсуждение}

Результаты моделирования приведены на рис. 3-9. Процесс коммутации состоит из ряда последовательных

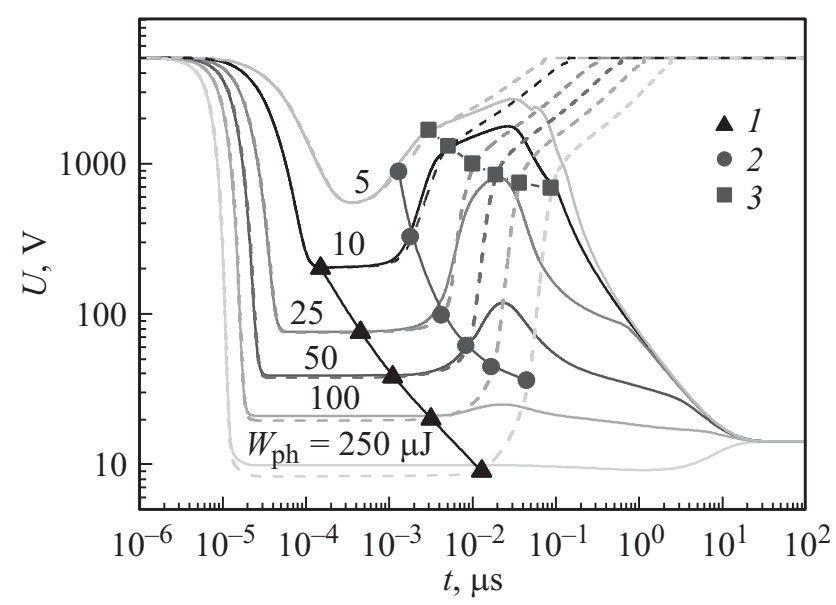

Рис. 3. Вольт-секундные характеристики $U(t)$ процессов коммутации фотодиодов (штриховые кривые) и фототиристоров (сплошные) при различных $W_{\mathrm{ph}}$. Символами отмечены моменты восстановления $p-v$-перехода $t_{0 p}(1)$, начала формирования ОПЗ $t_{s c}(2)$ и начала динамического лавинного пробоя $t_{\mathrm{av}}(3)$ фотодиода. 
этапов (см. рис. 3), которые сначала мы опишем для фотодиодов.

\section{1. Переключение в проводящее состояние}

На первом этапе управляющее излучение порождает неравновесные электроны и дырки с концентрациями $n(x, t), p(x, t)$ и через структуры начинает протекать ток проводимости с плотностью $j=q\left(n v_{n}+p v_{p}\right)$, где дрейфовые скорости $v_{n, p}=\bar{v}_{n, p} E /\left(E+\bar{E}_{n, p}\right), \bar{v}_{n, p}=\mu_{n, p} \bar{E}_{n, p}$, $\mu_{n, p}$ - низкополевые подвижности, $E-$ напряженность поля. Этот ток разряжает емкость структур $C_{D}=\varepsilon S_{D} / w_{0}$ и индуцирует ток нагрузки (см. рис. 4), равный [15]

$$
I_{L}(\theta)=S_{\mathrm{ph}} \int_{0}^{\theta} \bar{j}\left(\theta^{\prime}\right) \exp \left(\theta^{\prime}-\theta\right) d \theta^{\prime}
$$

где безразмерное время $\theta=t / \tau_{D}, \tau_{D}=R C_{D}, \bar{j}-$ усредненная по толщине $w_{0}=x_{n}-x_{p}$ истощенного слоя плотность тока электронов и дырок, $x_{p, n}-$ границы истощенной области. При этом падение напряжения на структурах $U=U_{0}-I_{L} R$ быстро уменьшается, как изображено на рис. 3 . Простую оценку $\bar{j}$ можно сделать для малых времен, когда еще $t v_{n, p} \ll \min \left(d, \kappa^{-1}\right), n \approx p$, $E>\bar{E}_{n, p} \sim 10$ кВ/см почти во всем истощенном слое и поэтому

$$
\bar{j}(t) \approx q\left(\bar{v}_{n}+\bar{v}_{p}\right) G_{0} w_{0}^{-1} \int_{x_{p}}^{x_{n}} F_{x}(x) d x \int_{0}^{t} F_{t}(t) d t^{\prime} .
$$

Подстановка (4) в (3) дает после интегрирования с учетом (1), (2) следуюшую формулу для тока нагрузки:

$$
\begin{gathered}
I_{L}(\theta) \approx I_{0}\left[1-e^{-\theta} \Phi\left(\theta, \tau_{d} / t_{\mathrm{ph}}\right)\right] \\
I_{0}=q \frac{W_{\mathrm{ph}}}{\hbar \omega} \frac{\bar{v}_{n}+\bar{v}_{p}}{w_{0}}\left(1-R_{\mathrm{ph}}\right) 2 e^{-\kappa d} \\
\times\left\{\operatorname{sh}\left[\kappa\left(d-x_{p}\right)\right]-\operatorname{sh}\left[\kappa\left(d-x_{n}\right)\right]\right\}, \\
\Phi(\theta, \gamma)=1+\frac{\sqrt{\pi}}{2 \gamma} \exp \left(\frac{1}{4 \gamma^{2}}\right) \\
\times\left[\operatorname{erf}\left(\gamma \theta-\frac{1}{2 \gamma}\right)+\operatorname{erf}\left(\frac{1}{2 \gamma}\right)\right] .
\end{gathered}
$$

Эта формула хорошо описывает зависимость $I_{L}\left(t / \tau_{D}\right)$ вплоть до значений $I_{L} \approx 0.7 U_{0} / R$ (см. рис. 4$)$, после чего она дает завышенные значения, так как не учитывает снижение дрейфовых скоростей вследствие уменьшения со временем напряженности поля до значений, меньших $\bar{E}_{n, p}$ (см. рис. 5). Тем не менее она позволяет оценить „инженерное“ время коммутации $t_{0.9}$, являющееся решением уравнения $I_{L}\left(t_{0.9} / \tau_{D}\right)=0.9 U_{0} / R$, занижая его

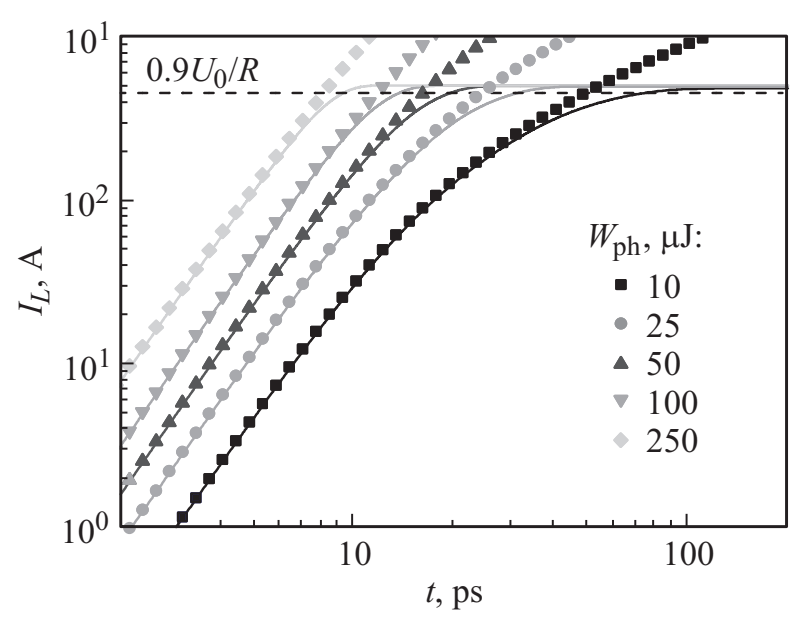

Pис. 4. Зависимости тока нагрузки от времени на первом этапе процесса коммутации фотодиода при различных энергиях импульса света $W_{\text {ph. }}$. Сплошные линии - результаты численного моделирования, точки -- расчет по формуле (5).

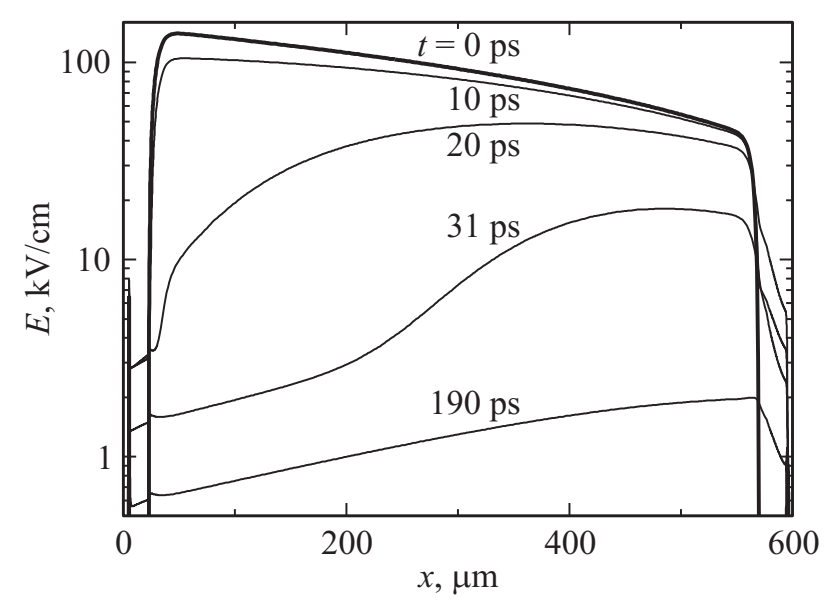

Рис. 5. Распределения электрического поля в фотодиоде при $W_{\text {ph }}=25$ мкДж в различные моменты времени. „Инженерное“ время коммутации $t_{0.9}=31$ пс.

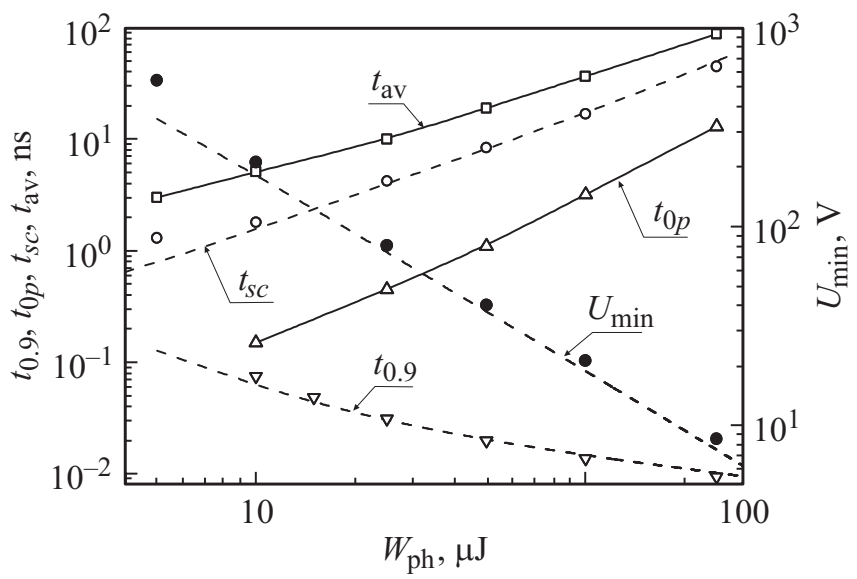

Рис. 6. Зависимости времен $t_{0.9}, t_{0 p}, t_{s c}, t_{\mathrm{av}}$ и напряжения $U_{\min }$ на фотодиоде от энергии импульса $W_{\mathrm{ph}}$. Точки - результаты численного моделирования, штриховые линии - расчет по формулам (6), (7) и (10). 


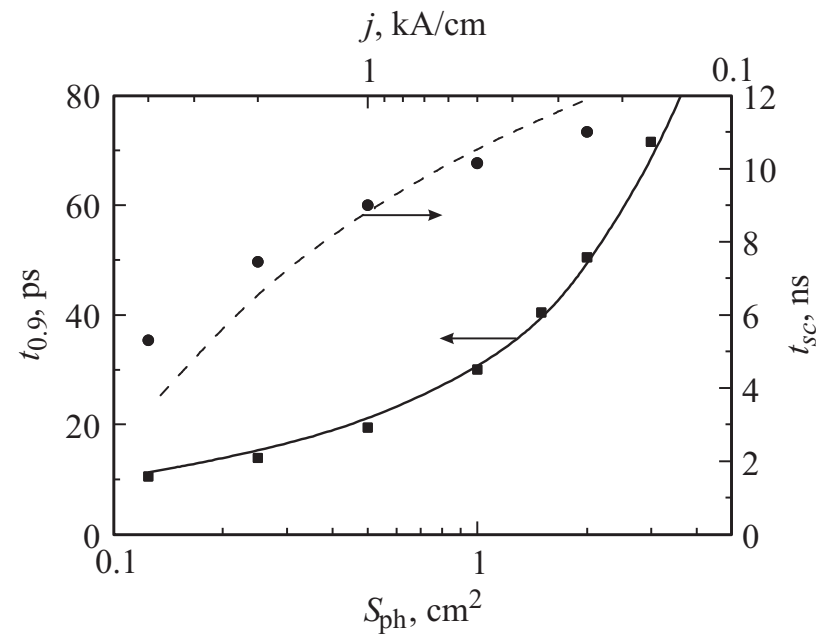

Рис. 7. Зависимости времен $t_{0.9}, t_{s c}$ фотодиодного коммутатора от $S_{\mathrm{ph}}$ при $W_{\mathrm{ph}}=50$ мкДж. Точки - результаты численного моделирования, линии - расчет по формулам (6) и (10).

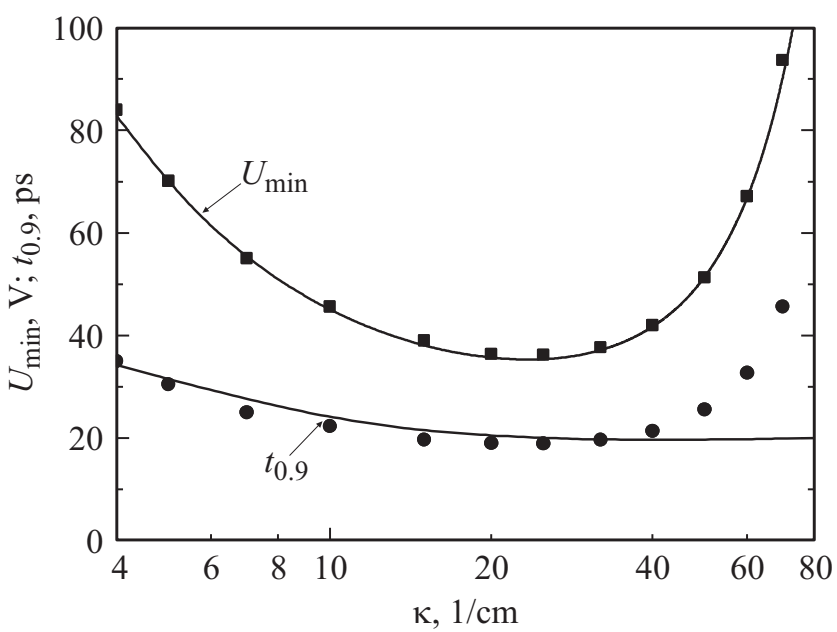

Рис. 8. Зависимости времени коммутации $t_{0.9}$ и напряжения $U_{\min }$ от коэффициента поглощения $\kappa$ для фотодиода при $W_{\mathrm{ph}}=50$ мкДж. Точки - результаты численного моделирования, линии - расчет по формулам (6) и (7).

на 20-30\%. Эту погрешность можно скомпенсировать, используя для расчета $t_{0.9}$ модифицированное уравнение

$$
I_{L}\left(\frac{4 t_{0.9}}{5 \tau_{D}}\right)=0.9 U_{0} / R
$$

Введение в (6) дополнительного множителя 4/5 обеспечивает описание зависимостей $t_{0.9}$ от $W_{\text {ph }}$ (см. рис. 6), $S_{\mathrm{ph}}$ (см. рис. 7) и $\kappa$ (см. рис. 8) с достаточной для практических целей точностью, если коэффициент поглощения $\kappa<40 \mathrm{~cm}^{-1}$. При бо́льших $\kappa$ распределение неравновесных носителей заряда в $v$-слое становится очень неоднородным, условие $E>\bar{E}_{n, p}$ применимости формулы (5) нарушается гораздо раньше и поэтому уравнение (6) дает сильно заниженные значения $t_{0.9}$, как показано на рис. 8 .
Следует отметить качественное различие распределений поля $E(x, t)$, изображенных на рис. 5, и полученных ранее в работе [15] для $p^{+}-v-n^{+}$-структур со ступенчатым легированием (см. рис. 5 в [15]). Оно состоит в том, что наличие относительно толстых и слабо легированных диффузионных слоев подавляет образование тонких областей с большой напряженностью поля на границах $v$-слоя и таким образом предотвращает преждевременное наступление динамического лавинного пробоя структур.

\section{2. Стадия высокой проводимости}

Второй этап наступает при $t>t_{0.9}$, когда напряженность поля становится меньше $\bar{E}_{n, p}$, напряжение на структуре $U(t)$ быстро уменьшается и достигает своего минимума

$$
U_{\min }=U_{0}\left[1+\left(1-R_{\mathrm{ph}}\right) \frac{W_{\mathrm{ph}}}{\hbar \omega} \frac{q\left(\mu_{n}+\mu_{p}\right) R_{L}}{d^{2}} \Psi(k d)\right]^{-1},
$$

где $\Psi(x)=2 x^{2} e^{-x}[\operatorname{arctg}(\operatorname{sh} x)]^{-1}$, ток нагрузки увеличивается и достигает максимума $I_{\max }=\left(U_{0}-U_{\min }\right) / R$, а ток через структуру $j S_{\mathrm{ph}}$, намного превосходящий $I_{L}$ во время первого этапа, быстро уменьшается и сравнивается с $I_{L}$. Формула $(7)$ хорошо описывает зависимости $U_{\min }$ от $W_{\text {ph }}$ (см. рис. 7 ) и $\kappa$ (см. рис. 8 ), но не учитывает электронно-дырочное рассеяние, которое ослабевает при снижении концентрации неравновесных носителей заряда. Вследствие этого напряжение $U_{\min }$ зависит еще и от $S_{\text {ph }}$, но очень слабо: оно уменьшается на $\sim 20 \%$ при увеличении $S_{\mathrm{ph}}$ в 20 раз и $W_{\mathrm{ph}}=$ const.

\section{3. Восстановление $p^{+}-n-$ и $n-n^{+}-$переходов}

Неравновесные электроны и дырки, накопленные в структурах во время освещения, начинают вытягиваться из приграничных областей. В фотодиодах это приводит к восстановлению ${ }^{2} p^{+}-p$ - и $n-n^{+}$-переходов, расположенных в плоскостях $x_{p}^{j}$ и $x_{n}^{j}$ в моменты $t=t_{0 p}$ и $t=t_{0 n}$. Аналитическая оценка $t_{0 p, 0 n}$ возможна в простейшем случае толстых однородно легированных $p^{+}$и $n^{+}$-слоев при низком уровне инжекции в них и высоком уровне инжекции в прилегающих $p$ - и $n$-слоях. Для времени восстановления $p^{+}-p$-перехода получается

$$
t_{0 p}=\frac{\pi}{4}\left[\frac{\mu_{n}+\mu_{p}}{\mu_{n}} \frac{n_{0}}{j}\right]\left(\sqrt{D}+\sqrt{D_{n}^{+}}\right)^{2},
$$

где $n_{0}=\kappa W_{\mathrm{ph}}\left(1-R_{\mathrm{ph}}\right) / \hbar \omega S_{\mathrm{ph}}, D_{n}^{+}-$коэффициент диффузии электронов в $p^{+}$-слое, $D=2 D_{n} D_{p} /\left(D_{n}+D_{p}\right)-$ амбиполярный коэффициент диффузии в слабо легированном Si. Формула (8) дает заниженное время $t_{0 p}$. Однако это практически не влияет на точность расчета $t_{s c}$ в

\footnotetext{
${ }^{2}$ Восстановлением этих переходов мы, как и в [18], называем процесс, в результате которого концентрация основных носителей заряда на границах $x=x_{p, n}^{j}$ практически сравнивается с $N_{a, d}$ соответственно.
} 
следующем разделе, так как „правильные“ значения $t_{0 p}$, полученные путем численного моделирования, примерно в 10 раз меньше $t_{s c}$ (см. рис. 6).

Восстановление завершается образованием крутых концентрационных фронтов, движущихся навстречу друг другу и отделяющих центральную плазменную область $x_{p}^{f}<x<x_{n}^{f}$ от областей $x<x_{p}^{f}, x>x_{n}^{f}$, практически свободных от неравновесных носителей заряда $[19,20]$.

\section{4. Формирование области пространственного заряда}

Концентрации легирующих примесей $N_{a, d}\left(x_{p, n}^{f}\right)$ на фронтах уменьшаются со временем. Поэтому при $t=t_{s c}$ в одной из областей $x<x_{p}^{f}, x>x_{n}^{f}$ нарушается условие квазинейтральности $[19,20]$. Если $\kappa$ не слишком велик, то сначала это происходит в области $x<x_{p}^{f}$ при $x_{p}^{f}=x_{p}^{s c}$, где $x_{p}^{s c}-$ решение уравнения [20]

$$
\left|N_{a}\left(\tilde{x}_{p}\right)\right|=\frac{1}{q}\left[\frac{j}{\bar{v}_{p}}+\sqrt{\frac{\varepsilon j}{\gamma \mu_{p} \lambda_{p}\left(\tilde{x}_{p}\right)}}\right],
$$

$\lambda_{p}^{-1}\left(\tilde{x}_{p}\right)=\partial \ln N_{a}\left(\tilde{x}_{p}\right) / \partial x, \gamma \sim 0.1$ - параметр, характеризующий степень нарушения нейтральности. Численное решение (9) позволяет вычислить $t_{s c}$ по формуле [20]

$$
t_{s c} \approx t_{0 p}+\frac{\mu_{n}+\mu_{p}}{\mu_{n}} \frac{n_{0}}{j}\left(x_{p}^{s c}-x_{p}^{j}\right) .
$$

При выводе (10) мы пренебрегали слабыми зависимостями тока $S_{\mathrm{ph}} j$ от времени при $t_{0 p}<t<t_{s c}$ (см. рис. 4) и концентрации неравновесных электронов перед фронтом от координаты (вследствие неравенства $\kappa x_{p}^{s c} \ll 1$ ), а также полагали, что $N_{a} \ll n_{0}$ в области $x_{p}^{j}<x<x_{p}^{s c}$. Последнее условие нарушается при малых $W_{\mathrm{ph}}$ и больших $S_{\mathrm{ph}}$, однако формула (10) неплохо описывает зависимости $t_{s c}$ от $W_{\mathrm{ph}}$ (см. рис. 6) и $S_{\mathrm{ph}}$ (см. рис. 7).

После нарушения нейтральности начинается третий этап, во время которого за пределами плазменной области образуются области пространственного заряда (ОП3). ОПЗ быстро расширяются до тех пор, пока вследствие роста напряженности поля в них не начинается ударная ионизация при $t=t_{\mathrm{av}}$ (см. рис. 9). Это приводит к сильному замедлению роста напряжения на структуре (см. рис. 3) [20] вплоть до полного восстановления ее блокирующей способности. Однако во время этого заключительного этапа реализуется режим двойной лавинной инжекции, которая может привести к шнурованию тока и разрушению структуры, наблюдавшемуся в ранней экспериментальной работе [12]. Это явление изучалось во многих работах (см. ссылки в обзорах [21,22]), однако в нашем случае оно может обладать рядом нетривиальных особенностей вследствие того, что скорость уменьшения тока в десятки раз превышает обычные значения. Анализу этих особенностей будет посвящена отдельная работа.
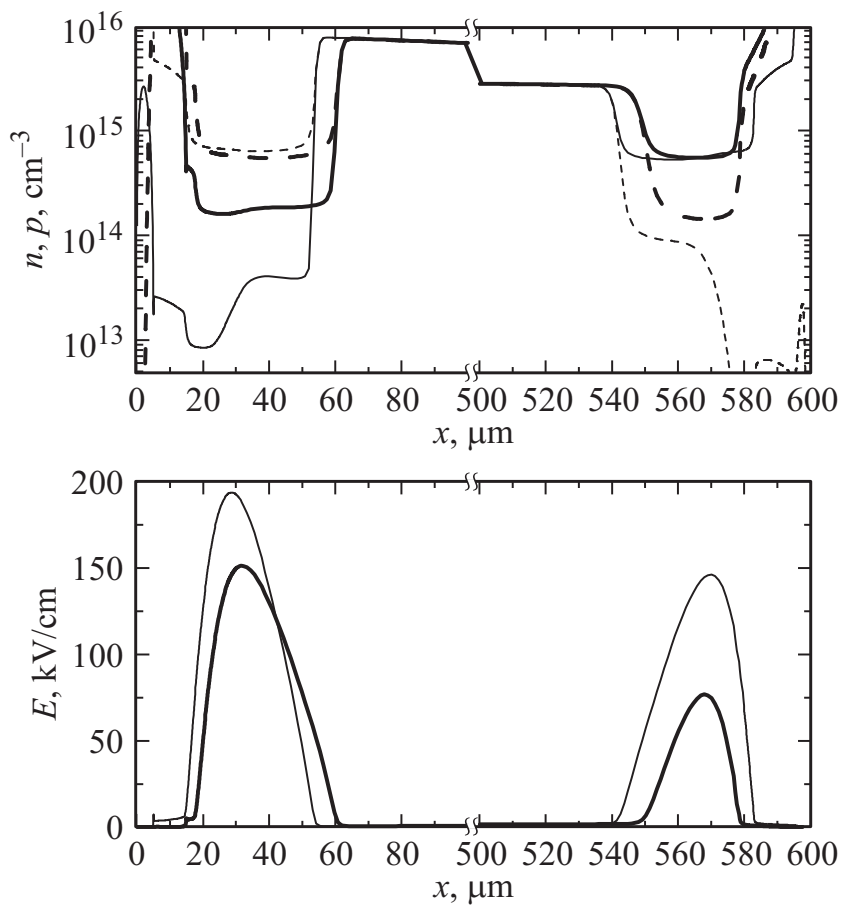

Рис. 9. Распределения электронов ( $n$, сплошные линии), дырок ( $p$, штриховые линии) и электрического поля $(E)$ в фотодиоде (тонкие линии) и фототиристоре (толстые линии) при $W_{\mathrm{ph}}=25$ мкДж в момент времени $t=t_{\mathrm{av}}=10 \mathrm{Hc}$.

\section{5. Особенности работы фототранзистора и фототиристора}

Во время первых двух этапов процессы коммутации фотодиода и фототранзистора практически совпадают. Различия возникают на третьем этапе. В фототранзисторе обычная инжекция дырок из прямосмещенного анодного $n-p^{+}$-перехода подавляет его восстановление и образование прианодной ОПЗ. Поэтому нарастание напряжения происходит чуть медленнее. На последнем этапе фототранзистор восстанавливает свою блокирующую способность так же, как и обычные биполярные коммутаторы типа GTO или IGBT [23], и может разрушиться по тем же причинам [23].

В фототиристоре, кроме того, добавляется обычная инжекция электронов из прямосмещенного катодного $n^{+}-p$-перехода. Однако она начинает проявляться с задержкой $t_{d} \sim 10 \mathrm{Hc}$, по порядку величины равной времени пролета через сильно легированную часть $p$-базы ${ }^{3}$ толщиной $t_{p}=8$ мкм и с концентрацией акцепторов $N_{a} \approx 2 \cdot 10^{17} \mathrm{~cm}^{-3}[24]$. Если $W_{\mathrm{ph}} \geq 100$ мкДж, то $t_{d}<t_{0 p}, p^{+}-p$-переход не успевает восстановиться и ОПЗ не возникает. В этом простейшем случае, рассмотренном в [14], фототиристор ведет себя как прямосмещенный диод, переходящий со временем в стационарное состояние. При меньших $W_{\mathrm{ph}}$ выполняется

\footnotetext{
3 Этот слой введен для повышения эффективности шунтировки, которая обеспечивает напряжение переключения более 5 кВ при $d U / d t \sim 1 \mathrm{\kappa B} / \mathrm{м \kappa с}$
} 
неравенство $t_{d}>t_{0 p}, p^{+}-p$-переход успевает восстановиться, ОПЗ возникает и начинает расширяться. Однако при $W_{\mathrm{ph}} \geq 20$ мкДж этот процесс подавляется инжекцией электронов, так что примерно через 20 нс напряжение на фототиристоре вновь начинает уменьшаться за счет обычного регенеративного механизма включения. Если же $W_{\mathrm{ph}}<20$ мкДж, то этот механизм также срабатывает, но только после кратковременной стадии лавинной инжекции. Отметим, что описанный выше немонотонный характер включения фототиристора наблюдался ранее в экспериментах $[4,6]$.

Автор благодарен С.Н. Юркову и Т.Т. Мнацаканову, предоставившим возможность провести моделирование процесса комммутации с помощью программы „Исследование“, и А.В. Горбатюку за интерес к работе и полезные обсуждения.

Работа выполнена при поддержке РФФИ (грант № 16-08-01292).

\section{Список литературы}

[1] И.В. Грехов, М.Е. Левинштейн, В.Г. Сергеев. ФТП, 8, 672 (1974).

[2] И.В. Грехов, М.Е. Левинштейн, В.Г. Сергеев. ФТП, 10, 345 (1976).

[3] O.S.F. Zucker, J.R. Long, V.I. Smith, D.J. Page, P.L. Hower. Appl. Phys. Lett., 29, 261 (1976).

[4] В.М. Волле, И.В. Грехов, А.Ф. Кардо-Сысоев, М.Е. Левинштейн, В.Г. Сергеев, И.Г. Чашников. Письма ЖТФ, 3 (20), 1077 (1977).

[5] И.В. Грехов, М.Е. Левинштейн, В.Г. Сергеев, И.Н. Яссиевич. ЖТФ, 49, 1013 (1979).

[6] В.М. Волле, В.М. Воронков, И.В. Грехов, М.Е. Левинштейн, В.Г. Сергеев, И.Г. Чашников. ЖТФ, 51 (2), 373 (1981).

[7] И.В. Грехов, В.Г. Сергеев, М.Е. Левинштейн, И.Г. Чашников, В.М. Волле, В.М. Воронков, И.Н. Яссиевич. Патент СССР № 730227. Опубл. 07.10.1982 г., Бюллетень № 37.

[8] D. Giorgi, P.K.L. Yu, J.R. Long, V.D. Lew, T. Navapanich, O.S.F. Zucker. J. Appl. Phys., 63, 930 (1988).

[9] J.H. Hur, P. Hadizad, S.G. Hummel, K.M. Dzurko, P.D. Dapkus H.R. Fetterman, M.A. Gundersen. IEEE Trans. Electron Dev., 37, 2520 (1990).

[10] H.D. Sanders, S.C. Glidden, D.M. Warnow. In: 2011 IEEE Pulsed Power Conference, (IEEE, 2011) p. 794.

[11] S.C. Glidden, H.D. Sanders. US Patent № 8, 461, 620 B2 (2013).

[12] http://www.ipgphotonics.com/category/4/Lasers

[13] И.В. Грехов, И.Н. Яссиевич. ФТП, 13, 1710 (1979).

[14] И.В. Грехов, И.Н. Яссиевич. ФТП, 14, 1747 (1980).

[15] А.С. Кюрегян. ФТП, 48, 1686 (2014).

[16] T.T. Mnatsakanov, I.L. Rostovtsev, N.I. Philatov. Solid-State Electron., 30, 579 (1987).

[17] M.A. Green. Solar Energy Mater. \& Solar Cells, 92, 1305 (2008).

[18] D. Lax, S.F. Neustadter. J. Appl. Phys., 25, 1148 (1954).

[19] H. Benda, F. Dannhauser. Solid-State Electron., 11, 1 (1968).

[20] И.В. Грехов, А.С. Кюрегян. ЖТФ, 75, 88 (2005).

[21] J. Lutz, R. Baburske. Microelectron. Reliab., 52, 475 (2012).
[22] H.-J. Schulze, F.-J. Niedernostheide, F. Pfirsch, R. Baburske. IEEE Trans. Electron Dev., 60, 551 (2013).

[23] А.В. Горбатюк, Д.В. Гусин, Б.В. Иванов. ФТП, 47, 373 (2013).

[24] J.L. Moll, I.M. Ross. Proc. IRE, 44, 72 (1956).

Редактор Л.В. Шаронова

\section{Power nano- and picosecond optoelectronic switches based on high-voltage silicon structures with $p-n$-junctions. I. Physics of switching process}

\section{A.S. Kyuregyan}

All-Russian Electrical Engineering Institute, 111250 Moscow, Russia

\begin{abstract}
Numerical simulation of switching process of high-voltage silicon photodiodes, phototransistors and photothyristors those triggered-on homogeneously over the area by picosecond laser pulses, has been performed for the first time. The analysis of results allowed to obtain „empirical“ relationship between key parameters of switches (energy of control pulses, a radiation absorption coefficient, the area of structures) and parameters characterizing the switching process in a circuit with the resistive load. For some of these relationship the approximate analytical formulas which seem to be quite adequate to the simulation results has been deduced. It is noted that distinctions between switching processes in structures of the three different types become apparent only at long duration of voltage pulses at a final stage when the blocking capability of photodiodes and phototransistors is recovered.
\end{abstract}

